\title{
MODELO MOLECULAR DE CARBONO ATIVADO PARA ADSORÇÃO DE COMPOSTOS SULFURADOS
}

\author{
F. S. P. DANTAS ${ }^{1}$, V. A. M. GOMES ${ }^{1}$, G. M. MAGALHÃES ${ }^{1}$, S. M. P. LUCENA ${ }^{1}$ \\ ${ }^{1}$ Universidade Federal do Ceará, Departamento de Engenharia Química. \\ e-mail: francisco.s.p.dantas@gmail.com
}

\begin{abstract}
RESUMO - Carbono ativado é um adsorvente amplamente usado para purificação de diversas correntes na indústria petroquímica, porém sua estrutura amorfa dificulta a elaboração de modelos apropriados. Este trabalho foi realizado com o intuito de propor modelos computacionais para sistemas de adsorção contendo compostos aromáticos sulfurados e carbono ativado. Foram utilizados três poros típicos das distribuições de poros encontrados em carbonos ativados $(8,9 \AA$, 18,5 e 30,9 ̊). Para os adsorbatos, foram propostos novos parâmetros para modelar as moléculas de benzotiofeno e de dibenzotiofeno. Simulações foram realizadas utilizando o método computacional de Monte Carlo. Isotermas de adsorção foram geradas à 393 e $453 \mathrm{~K}$, estas mostram que, para o poro menor, a adsorção ocorre em baixas pressões quando comparada com as isotermas dos poros maiores. O poro de $18,5 \AA$ apresentou a melhor relação entre quantidade adsorvida e calor de adsorção. Considerações sobre a distribuição de poros mais eficiente para a captura de aromáticos sulfurados são também apresentadas.
\end{abstract}

\section{INTRODUÇÃO}

Grandes quantidades de compostos sulfurados com concentrações variando entre 300 e 500 ppm em peso estão contidos nas composições da gasolina comercial e do diesel. Tais compostos podem causar muita poluição ambiental por conta dos produtos gerados da sua combustão, espécies do tipo SOx. Compostos sulfurados também podem causar corrosão severa de equipamentos e reatores durante as etapas de processamento de petróleo. A remoção de compostos sulfurados de derivados de petróleo é uma prática muito exigida atualmente e têm se tornado uma importante operação unitária em refinamento de petróleo.

Unidades de hidrodessulfurização (HDS) são utilizadas para redução de enxofre dos combustíveis. Este tratamento consiste em tratar cataliticamente compostos sulfurados com hidrogênio e convertê-los em $\mathrm{H}_{2} \mathrm{~S}$ e hidrocarbonetos. Estes processos removem eficientemente compostos sulfurados como tióis, sulfetos e dissulfetos, porém não são efetivos na remoção de tiofeno e seus derivados aromáticos e, por isso, o HDS apresenta sérias limitações para atingir os novos limites de concentração de compostos sulfurados da ordem de $10 \mathrm{ppm}$, além de ser um processo de alto custo. Tais fatores trazem condições favoráveis de competição para os processos de adsorção. O sucesso das tecnologias de adsorção dependem do custo dos adsorventes, da capacidade de adsorção e da seletividade com relação ao composto que deve ser removido. 


\section{MODELOS E MÉTODOS}

Para o estudo da adsorção foram definidos três tamanhos de poros muito comuns nas estruturas do adsorvente, o carbono ativado (poros: 8,9 $\AA, 18,5 \AA$ e $30,9 \AA$ ); e para o adsorbato, foram utilizados modelos para as moléculas de benzotiofeno e dibenzotiofeno. Valores de parâmetros de campo de força e cargas foram escolhidos de acordo com dados encontrados na literatura de forma a representar as características físicas e químicas do sistema real.

\subsection{Modelo Matemático}

As interações entre as moléculas dentro do sistema foram modeladas segundo a equação de energia potencial de Lennard-Jones em seu formato LJ_12_6, acrescentando a parcela do efeito eletrostático, descritas na Equação 1.

$$
U\left(r_{i a j b}\right)=4 \varepsilon\left[\left(\frac{\sigma}{r_{i a j b}}\right)^{12}-\left(\frac{\sigma}{r_{i a j b}}\right)^{6}\right]+\frac{q_{i a} q_{j b}}{r_{i a j b}}
$$

Onde $\varepsilon$ representa a energia mínima da curva, $\sigma$ é a distância mínima na qual a energia de interação é nula, $r_{i a j b}$ é a distância entre os centros moleculares $i_{a}$ e jb, e $q_{i a}$ e $q_{j b}$ são as cargas dos átomos.

Para calcular os parâmetros $\sigma$ e $\varepsilon$ entre átomos de diferentes moléculas, as regras de combinação de Lorentz-Berthelot podem ser utilizadas. Mostradas nas Equações 2 e 3.

$$
\begin{aligned}
\varepsilon_{i j} & =\sqrt{\varepsilon_{i j} \varepsilon_{i j}} \\
\sigma_{i j} & =\frac{\sigma_{i j}+\sigma_{i j}}{2}
\end{aligned}
$$

Benzotiofeno: Combinou-se o modelo de tiofeno descrito por Ju et al. (2006) com o modelo de benzeno de Contreras-Camacho et al. (2004) (Figura 1a). No modelo de Ju et al. (2006) o tiofeno consiste de uma molécula rígida e plana com unidades $\mathrm{C}-\mathrm{H}$ representadas no sistema de átomos unitários. $\mathrm{O}$ anel tem movimentos de torsão limitados pela sua flexibilidade. Os comprimentos de ligação C - C e S - C são fixados em 1,4 Å e 1,72 Å, respectivamente. A molécula de benzeno tem seus átomos representados explicitamente com exceção dos que são comuns a molécula de tiofeno.

Dibenzotiofeno: O modelo utilizado também baseou-se nos modelos individuais de tiofeno e benzeno descritos no modelo do benzotiofeno e está ilustrado na figura $1 \mathrm{~b}$, bem como as cargas de cada átomo, também podem ser visualizadas. 

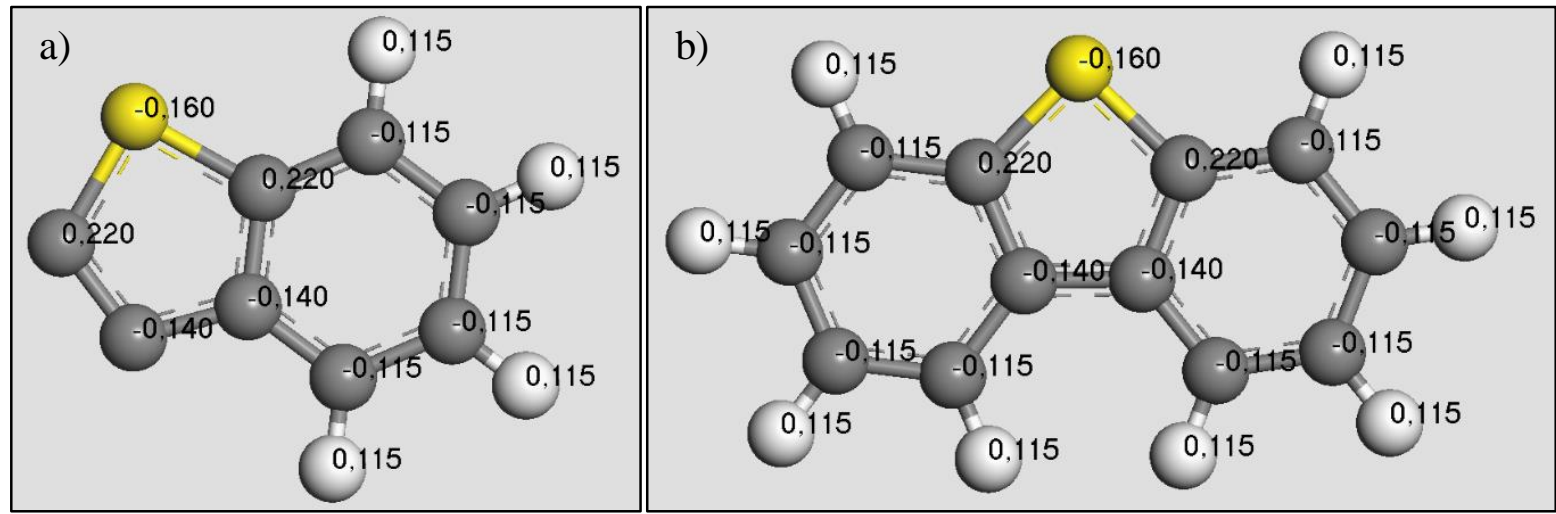

Figura 1 - Modelo proposto para a molécula de benzotiofeno (a) e de dibenzotiofeno (b). Os valores das cargas utilizadas estão também indicados na figura.

Carbono Ativado: O modelo de placas paralelas para os poros de carbono ativado foi retirado de Lucena et al. (2010) com paredes compostas por duas camada de grafeno. A carga do átomo de carbono encontrado na estrutura do carbono ativado é zero. Três tipos de células de simulação foram utilizadas, variando o tamanho de poro do sistema que é equivalente à distância entre as placas mais internas do modelo, os tamanhos de poro utilizados foram: 8,9, 18,5 e $30,9 \AA$ A. Três tamanhos diferentes de poros foram utilizados de maneira a representar o comportamento da adsorção nestes materiais amorfos. Uma ilustração da estrutura pode ser visualizada na figura 2.

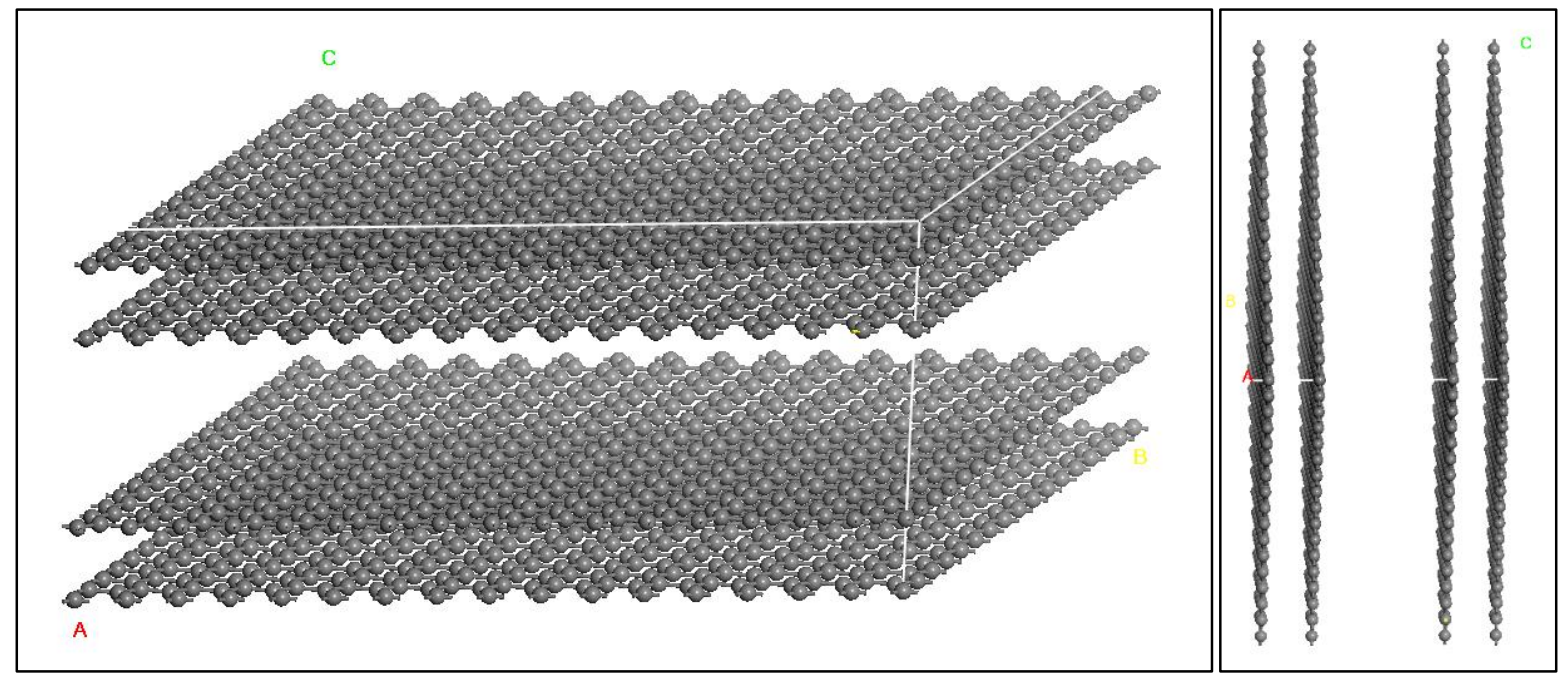

Figura 2 - Representações geométricas da estrutura do carbono ativado de poro 8,9 A.

Parâmetros: Os parâmetros de campo de força para o sistema adsorvente/adsorbato podem ser encontrados na Tabela 1 . Onde $\mathrm{D}_{0}$ e $\mathrm{R}_{0}$ se correlacionam diretamente e respectivamente com $\sigma$ e $\varepsilon$, parâmetros da equação de Lennard-Jones (Equação 1). Os parâmetros mostrados na Tabela 1 são parâmetros de interação individuais. $\mathrm{C}_{\mathrm{c}}$ é a classificação que foi dada aos átomos de carbono pertencentes à estrutura do adsorvente. 
Tabela 1 - Parâmetros de campo de força individuais.

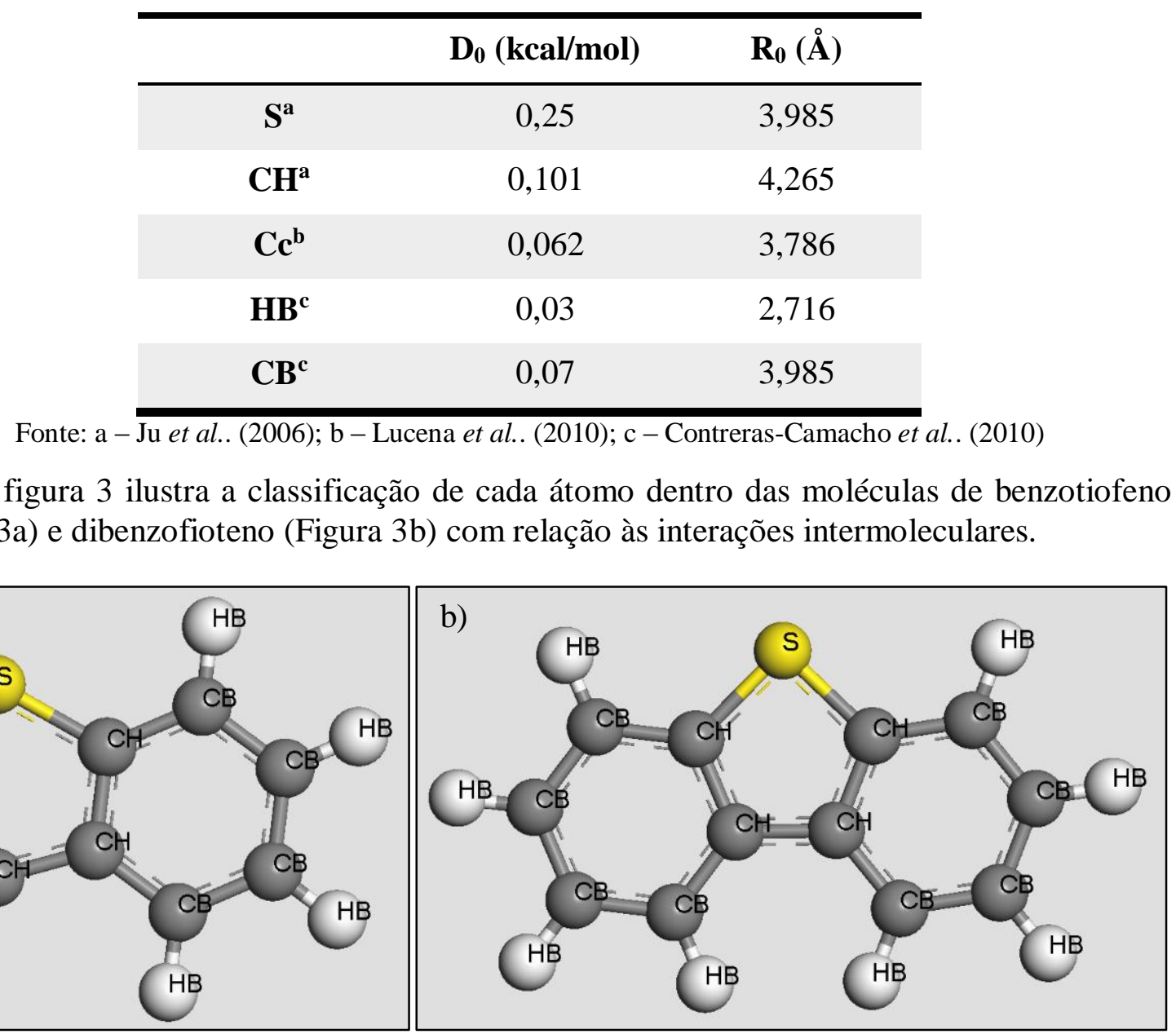

Figura 3 - Representações geométricas de benzotiofeno (a) e de dibenzotiofeno (b).

O método computacional de Monte Carlo no ensemble Grande Canônico (GCMC) foi utilizado para cálculo de isotermas de adsorção a 393 K e $453 \mathrm{~K}$. Para cada ponto de cada isoterma de adsorção foram utilizados um milhão e meio de iterações, garantindo que o sistema atinja todos os estados acessíveis possíveis. Cada curva que representa uma isoterma de adsorção é composta por 50 pontos simulados e as faixas de pressão foram escolhidas levando em consideração as pressões de vapor dos adsorbatos. Para limitar os cálculos de interações entre os átomos, foi configurado um raio de atuação da força dessas interações, ou cutoff, de valor igual a $12,5 \AA$, de forma que as interações só fossem calculadas até essa distância, reduzindo o tempo computacional de cada simulação.

\section{DISCUSSÃO DE RESULTADOS}

Utilizando os modelos descritos anteriormente, dois sistemas de adsorção foram estudados, um contendo benzotiofeno e carbono ativado e outro contendo dibenzotiofeno e carbono ativado. Isotermas de adsorção foram simuladas a $393 \mathrm{~K}\left(120^{\circ} \mathrm{C}\right)$ e a $453 \mathrm{~K}\left(180{ }^{\circ} \mathrm{C}\right)$. 


\subsection{Adsorção de Benzotiofeno em Carbono Ativado}

Isotermas de adsorção de benzotiofeno em carbono ativado foram simuladas de $10^{-8} \mathrm{kPa}$ até $5 \mathrm{kPa}$ a $393 \mathrm{~K}$ e de $10^{-8} \mathrm{kPa}$ até $36 \mathrm{kPa}$ a $453 \mathrm{~K}$, para cada um dos três poros. Isotermas simuladas a $393 \mathrm{~K}$ são mostradas na Figura 4, enquanto as isotermas simuladas a $453 \mathrm{~K}$ se encontram na Figura 5.

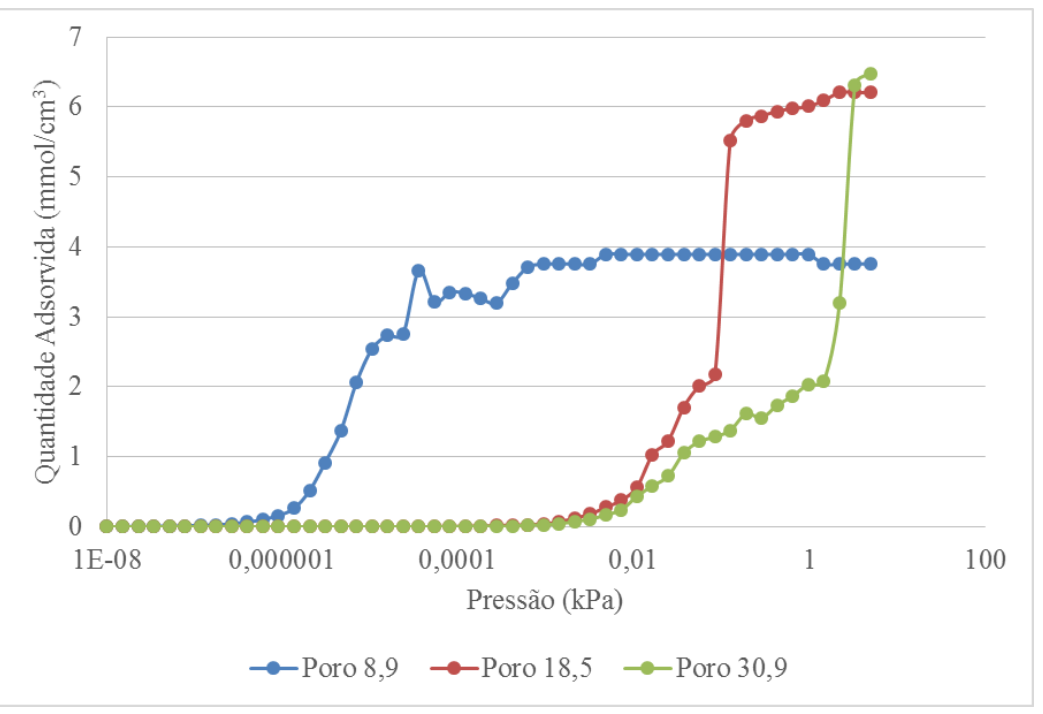

Figura 4 - Isotermas de adsorção de benzotiofeno em carbono ativado a 393 K.

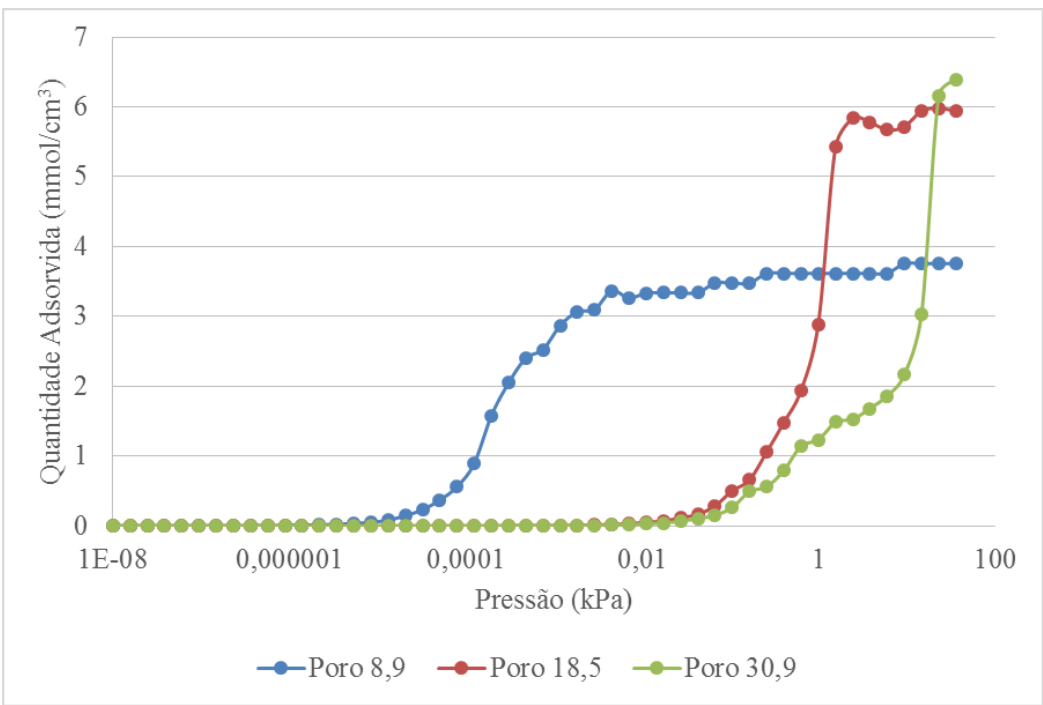

Figura 5 - Isotermas de adsorção de benzotiofeno em carbono ativado a $453 \mathrm{~K}$.

As figuras 4 e 5 indicam que o poro maior, de 30,9 Å, têm capacidade de adsorção equivalente ao poro de 18,5 , no entanto, o calor de adsorção do poro de 18,5 (25,4 kcal/mol) é maior que o do poro de $30,9(22,8 \mathrm{kcal} / \mathrm{mol})$, o que faz com que o preenchimento do poro de 30,9 ocorra mais tardiamente sendo atingido somente nas pressões maiores, nas proximidades das pressões de vapor do adsorbato. O poro de 8,9 é rapidamente preenchido apresentando capacidade de adsorção bem inferior aos outros poros. O efeito da temperatura, neste sistema, 
indica que menores quantidades são adsorvidas para maiores temperaturas à uma dada pressão, porém o perfil relativo entre os poros não tem mudança significativa. Com relação à capacidade adsortiva, a temperatura não tem efeito considerável em nenhum dos poros.

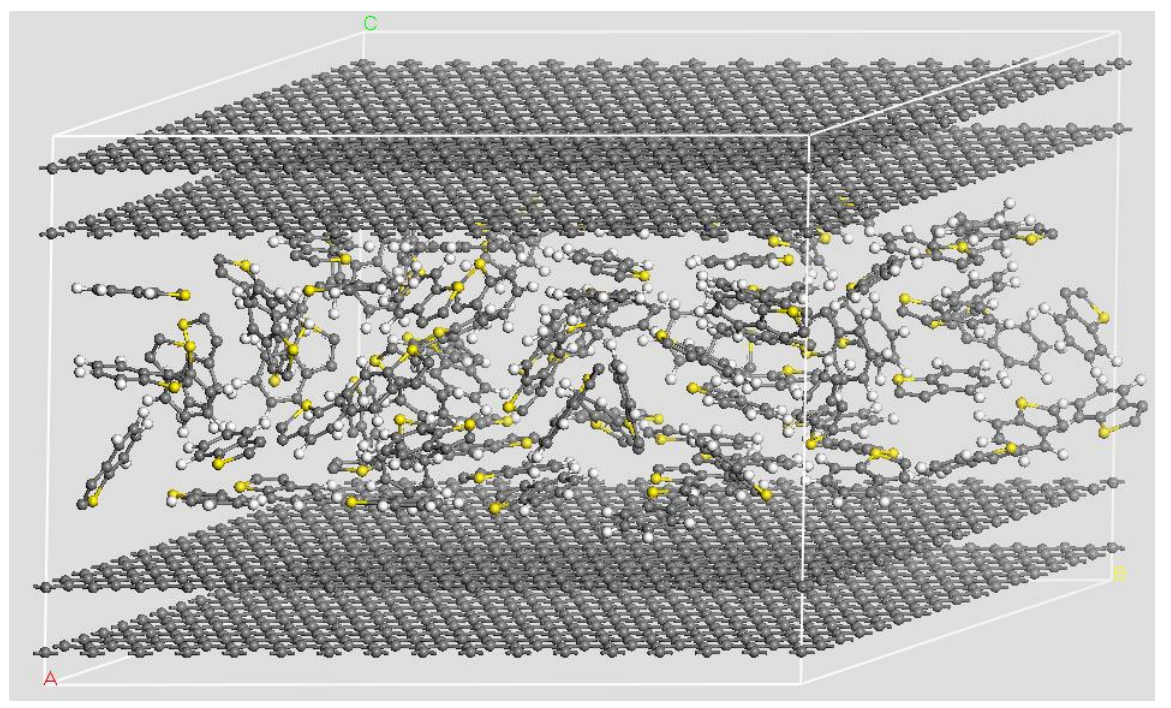

Figura 6 - Ilustração da estrutura de carbono ativado com poro de 18,9 Å após a adsorção de benzotiofeno.

A figura 6 ilustra o material poroso, carbono ativado com poro $18,9 \AA$ A, com moléculas de adsorbato inseridas dentro do poro pelo processo de adsorção.

\subsection{Adsorção de Dibenzotiofeno em Carbono Ativado}

Isotermas de adsorção de dibenzotiofeno em carbono ativado foram simuladas de $10^{-10}$ $\mathrm{kPa}$ até $0,1 \mathrm{kPa}$ a $393 \mathrm{~K}$ e de $10^{-10} \mathrm{kPa}$ até $1,4 \mathrm{kPa}$ a $453 \mathrm{~K}$, para cada um dos três poros. Isotermas simuladas a $393 \mathrm{~K}$ são mostradas na Figura 7, enquanto as isotermas simuladas a 453 K se encontram na Figura 8.

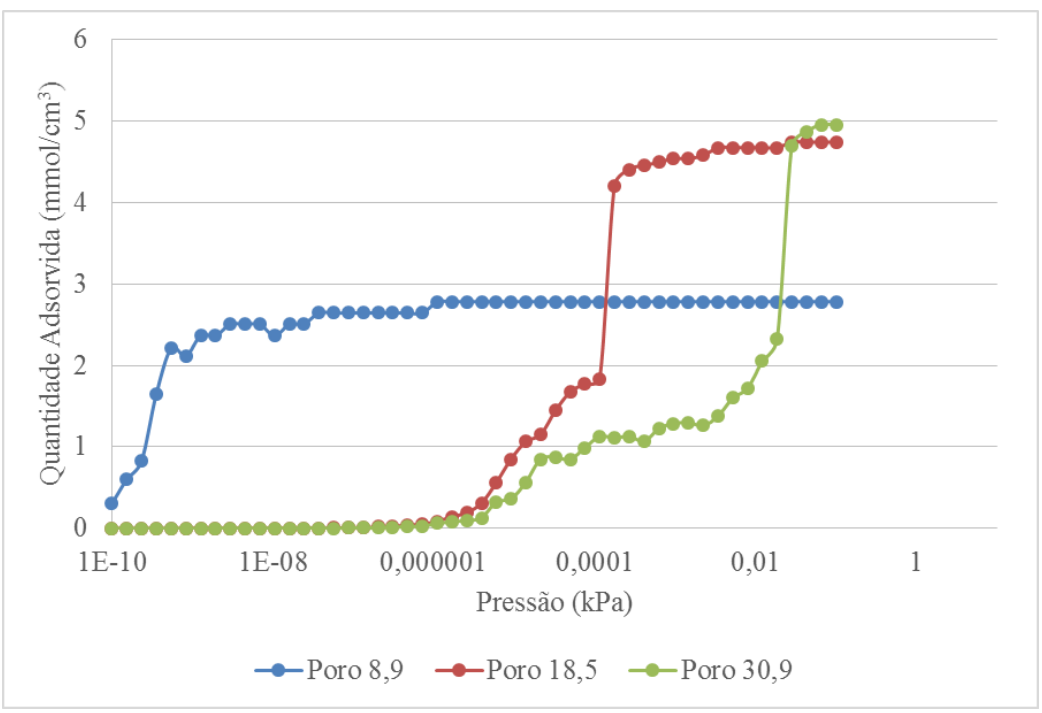

Figura 7 - Isotermas de adsorção de benzotiofeno em carbono ativado a 393 K. 


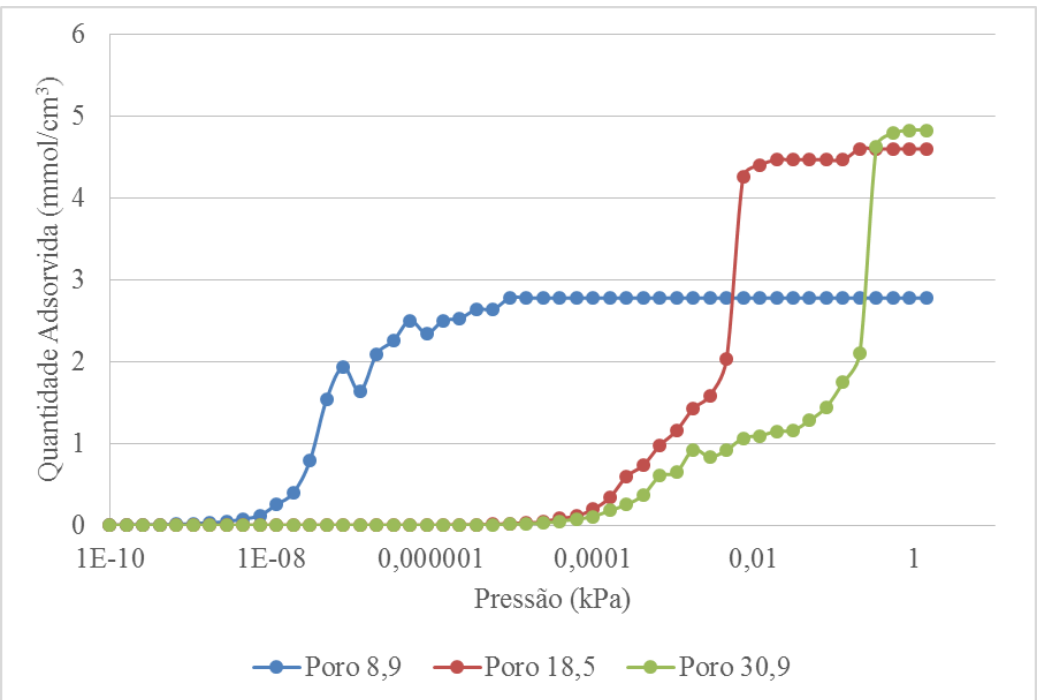

Figura 8 - Isotermas de adsorção de benzotiofeno em carbono ativado a $453 \mathrm{~K}$.

As isotermas de adsorção de dibenzotiofeno, Figuras 7 e 8, têm um comportamento similar ao das isotermas de adsorção de benzotiofeno, figuras 4 e 5, porém a adsorção ocorre em menores pressões e as capacidades adsortivas são menores. Os calores de adsorção são maiores $(37,1 \mathrm{kcal} / \mathrm{mol}$ e $33,9 \mathrm{kcal} / \mathrm{mol}$ para os poros de 18,5 e 30,9 respectivamente). Estas característica refletem o maior tamanho da molécula. A temperatura também não tem efeito na capacidade adsortiva neste sistema.

Supondo a utilização prática de carbono ativado para esta finalidade o material deve maximizar seus poros na faixa dos 18,5 ̊̊ já próximo do limite entre micro e mesoporos.

\section{CONCLUSÕES}

Com o auxílio da modelagem molecular, utilizando parâmetros de campo de força e de carga que ainda não tiveram utilização nos sistemas estudados, isotermas de adsorção foram simuladas. As isotermas de adsorção para o poro de $8,9 \AA$ é do tipo I, diferenciando-a das demais, que são do tipo II, isso indica preenchimento de poro em intervalo de pressão definido, e resultando em maiores capacidades adsortivas. $\mathrm{O}$ fato de a molécula de dibenzotiofeno ter um anel a mais indica também que menos moléculas são adsorvidas, num mesmo espaço, quando comparado ao benzotiofeno. Tamanho de poro $(18,5 \AA)$ e sua respectiva pressão de preenchimento podem ser utilizadas para auxiliar no dimensionamento de um carbono ativado otimizado para a adsorção de aromáticos sulfurados. 


\section{REFERENCIAS BIBLIOGRÁFICAS}

Contreras-Camacho, R. O.; Ungerer, P.; Boutin, A.; Mackie, A. D. Optimized Intermolecular Potential for Aromatic Hydrocarbons Based on Anisotropic United Atoms. 1. Benzene. J. Phys. Chem. 108 (37), 14109-14114, 2004.

Di Lella, A.; Desbiens, N.; Boutin, A.; Demachy, I. Molecular Simulation Studies of Water Physisorption in Zeolites. J. Phys. Chem. v. 47, p. 1238-1244, 2008.

Faro Jr., A. C. Sulfetos e os hidrotratamentos. Curso de Catálise-Catalisadores e Processos Catalíticos, IBP, São Paulo, 1994.

Fitch, A. N.; Jobic, H.; Renouprez, A. Localization of Benzene In Sodium-Y Zeolite by Powder Neutron Diffraction. J. Phys. Chem., 90 (1986), 1311-1318.

Ju, S.-G., Zeng, Y.-P., Xing, W.-H., Chen, C.-L. Computer simulation of the adsorption of thiophene in all-silica Y and Na-Y. Langmuir, 22 (2006), 8353-8358.

Lucena, S.M.P; Paiva, C.A.S; Silvino, P.F.G; Azevedo, D.C.S; Cavalcante Jr, C.L. The effect of heterogeneity in the randomly etched graphite model for carbon pore size characterization. Carbon , 48 (2010), $2554-2565$.

Sing, K. S. W.; Everett, D. H.; Haul, R. A. W.; Moscou, L.; Pierotti, R. A.; Rouquerol, J.; Siemieniewska, T. Reporting Physisorption Data for Gas/Solid Systems with Special Reference to the Determination of Surface Area and Porosity, Pure and Applied Chemistry, 57 (1985), 603-619.

Yang, R. T. Adsorbents: Fundamentals and Applications. New Jersey: Ed. John Wiley \& Sons, Inc., 2003. 